\title{
Das soziale Europa aus der Perspektive der deutschen Gerichtsbarkeit
}

\author{
Ulrich Koch
}

Erste These

Zweite These

1. Vorbemerkung

2. Fehlende Verankerung in den gemeinsamen Verfassungstraditionen der Mitgliedstaaten

3. Mögliche Berücksichtigung des nationalen Rechts bei der gemeinschaftsrechtlichen Ausgestaltung des Streikrechts

4. Widersprüche in der Ausgestaltung des Streikrechts durch den europäischen Gerichtshof gegenüber dem nationalem Recht

5. Die Abwägung zwischen Grundfreiheiten und Grundrechten

6. Die Prüfung unter dem Gesichtspunkt der Verhältnismäßigkeit 51

7. Die Konkurrenzsituation zwischen Gemeinschaftsrecht und nationalem Recht 52

Dritte These

1. Die Entscheidung Werhof

2. Der Super-GAU - der Konflikt zwischen Gemeinschafts- und nationalem Verfassungsrecht

Mit meinen Ausführungen werde ich nicht in das teilweise in der Politik und bei den Verbänden zu vernehmende Klagelied darüber einstimmen, dass der Europäische Gerichtshof einem Sozialdumping Vorschub leistet. Eine solche Bewertung der Rechtsprechung des Gerichtshofs, noch dazu im Rahmen dieser Veranstaltung, steht mir nicht zu. Ich werde mich im Folgenden auf drei Themen mit einem Bezug zu meiner richterlichen Tätigkeit beschränken, die ich auch wegen der mir zur Verfügung stehenden Zeit nur kurz anreißen kann, von denen ich aber hoffe, dass sie interessant genug sind, von Ihnen in der Diskussion aufgegriffen zu werden. ${ }^{1}$

Der Anwendungsvorrang des Gemeinschaftsrechts eröffnet der deutschen Arbeitsgerichtsbarkeit die Möglichkeit, das nationale Recht und seine vermeintlich nicht mehr zeitgemäßen Regelungen unbeachtet zu lassen. Kommt es aber zu einem Nebeneinander

1 Auf Grund der mir zur Verfügung stehenden Zeit sind lediglich die Ausführungen zur Zweiten These - und diese auch nur in einer verkürzten Form - vorgetragen worden. 
besser noch - zu einem Miteinander von Gemeinschaftsrecht und nationalem Recht werden sowohl der Europäische Gerichtshof wie auch die deutschen Arbeitsgerichte immer wieder vor neue Herausforderungen gestellt. Über drei aus meiner Sicht aktuelle Bauvorhaben im Rahmen der Großbaustelle „Gemeinschaftsrecht und Arbeitsgerichtsbarkeit" möchte ich gerne sprechen.

Es geht zunächst um die Frage der Anwendbarkeit der allgemeinen Grundsätze des Gemeinschaftsrechts im nationalen Recht. Anschließend werde ich im Bereich des kollektiven Rechts auf mögliche Friktionen zwischen den beiden Rechtsquellen eingehen und mit Ausführungen zu zwei aktuellen Konfliktfällen zwischen dem Gemeinschaftsrecht und dem nationalen Recht schließen. Den Schwerpunkt der Betrachtungen werden dabei die Entscheidungen des Gerichtshofs in den Rechtssachen Mangold, Viking und Werhof bilden. Die Urteile in den Verfahren Rüffert und teilweise auch Laval mögen zwar für die Politik einen Handlungsbedarf begründen, sie haben aber - anders als die erstgenannten Entscheidungen - für die Gerichtsbarkeit keine herausragende Wirkung.

\section{Erste These}

Es fehlen präzise Kriterien, nach denen ein nationales Gericht beurteilen kann, ob ein allgemeiner Grundsatz des Gemeinschaftsrechts auf den von ihm zu beurteilenden Sachverhalt anzuwenden ist.

Ausgangspunkt für diese These ist der Sachverhalt, der der Entscheidung des Gerichtshofs in der Rechtssache Mangold zugrunde lag. Zwei offenbar gut miteinander bekannte Rechtsanwälte hatten einen befristeten Arbeitsvertrag geschlossen und seine Rechtfertigung ausschließlich auf $\S 14$ Abs. 3 Satz 4 TzBfG in der bis zum 30. April 2007 geltenden Fassung gestützt, und damit auf eine Vorschrift, die sie beide für unwirksam hielten. Das Arbeitsgericht München hatte zumindest Zweifel an der Vereinbarkeit von $\S 14$ Abs. 3 TzBfG mit dem Gemeinschaftsrecht und legte diese Frage dem Gerichtshof im Rahmen eines Vorabentscheidungsverfahrens vor.

Der Gerichtshof hat auf den Sachverhalt, in der es ausschließlich um die Rechtfertigung einer sachgrundlosen Befristung zwischen zwei Privatpersonen ging, den primärrechtlichen Grundsatz der Gleichbehandlung in Beschäftigung und Beruf herangezogen, der Diskriminierungen in Hinblick auf das Alter entgegensteht. Der Gerichtshof hat sich bei seinen Aussagen zum Anwendungsbereich des Gemeinschaftsrechts auf die Feststellung beschränkt, dass die in $\S 14$ Abs. 3 Satz 4 TzBfG aF enthaltene Regelung in den Geltungsbereich des Gemeinschaftsrechts fällt, weil der nationale Gesetzgeber mit dem TzBfG die Vorgaben der Richtlinie 1999/70 umsetzen wollte. Der Gerichtshof hat dazu weiter ausgeführt, dass damit alle nationalen Maßnahmen erfasst seien, die die Erreichung des mit der (Befristungs-)Richtlinie verfolgten Zieles gewährleisten sollen, ein- 
schließlich derjenigen, mit denen nach der eigentlichen Umsetzung die bereits erlassenen nationalen Rechtsvorschriften ergänzt oder geändert werden.

Die Befristungsrichtlinie dient aber nur dazu,

- durch die Anwendung des Grundsatzes der Nichtdiskriminierung die Qualität befristeter Arbeitsverhältnisse zu verbessern;

- einen Rahmen zu schaffen, der den Missbrauch durch aufeinander folgende befristete Arbeitsverträge oder -verhältnisse verhindert.

Keines der beiden Ziele steht mit dem in der Entscheidung Mangold behandelten Sachverhalt in Beziehung. Bei dem zur Prüfung gestellten befristeten Arbeitsvertrag handelte es sich um den erstmaligen Abschluss eines befristeten Arbeitsvertrags, so dass ein Missbrauch durch aufeinander folgende befristete Arbeitsverträge ausgeschlossen war. Auch eine Diskriminierung von Herrn Mangold gegenüber einem unbefristet beschäftigten Arbeitnehmer ist nach dem mitgeteilten Sachverhalt nicht geltend gemacht worden. Die einzige Verbindung, die ich sehe, ist der Abschluss eines befristeten Arbeitsvertrags, dessen Rechtfertigung sich nach den Bestimmungen des TzBfG bestimmte. ${ }^{2}$

Wie hätte ein nationales Gericht bis zum Ablauf der Umsetzungsfrist der Gleichbehandlungsrichtlinie entscheiden müssen, wenn die Bundesrepublik innerhalb der Umsetzungsfrist der Gleichbehandlungsrichtlinie $\S 1$ des Kündigungsschutzgesetzes um einen Absatz ergänzt hätte, nach dem für die Zeit von drei Jahren unbefristet eingestellte Arbeitnehmer, die das 52. Lebensjahr vollendet haben, erst nach Ablauf von 24 Monaten Kündigungsschutz erwerben? Wäre eine solche Regelung auch in den Anwendungsbereich des Gemeinschaftsrechts gefallen?

\section{Zweite These}

Der Einfluss des nationalen Rechts auf Arbeitskämpfe mit Gemeinschaftsbezug ist für die deutschen Arbeitsgerichte durch die Entscheidung des Europäischen Gerichtshofs nur teilweise geklärt.

Der Europäische Gerichtshof hat in der Entscheidung in der Rechtssache Viking das Recht auf Durchführung einer kollektiven Maßnahme einschließlich des Streikrechts als Grundrecht anerkannt, welches fester Bestandteil der allgemeinen Grundsätze des Gemeinschaftsrechts sei. ${ }^{3}$ Ich werde nachfolgend darauf eingehen, welche Vorgaben diese

2 Zweifel an der Reichweite des Grundsatzes der Gleichbehandlung in Beschäftigung und Beruf haben den Dritten Senat des BAG zu einem Vorabentscheidungsersuchen veranlasst, BAG vom 27. Juni 2006, 3 AZR 352/05 (A), BAGE 118, 340 = AP BetrAVG § 1b Nr. 6 = EzA Richtlinie 2000/78 EG-Vertrag 1999 Nr. 2, Rn. 29.

3 EuGH vom 11. Dezember 2007, C-438/05 (Viking), EzA GG Art. 9 Arbeitskampf Nr. 141 = NZA 2008, 124, Rn. 44. 
und die nachfolgende Entscheidung Laval für die deutschen Arbeitsgerichte enthält und ob dieses „Rüstzeug“ für eine Streitentscheidung ausreichend bemessen ist.

\section{Vorbemerkung}

Die Anerkennung eines Grundrechts auf kollektive Maßnahmen einschließlich des Streikrechts ist meines Erachtens zu begrüßen. Dies gilt aber nur eingeschränkt für seine dogmatische Herleitung und die inhaltliche Ausgestaltung des Grundrechts. Allerdings können die genanten Entscheidungen wegen der Besonderheiten in den zugrunde liegenden Sachverhalten wohl nur ein erster Fingerzeig auf die zukünftige gemeinschaftsrechtliche Ausgestaltung des Arbeitskampfrechts sein. In beiden Entscheidungen ging es nicht um einem gewerkschaftlich getragenen Streik von Arbeitnehmern gegen ihren eigenen Arbeitgeber zur Verbesserung ihrer Arbeitsbedingungen. Bei den Kollektivmaßnahmen handelte es sich ausschließlich oder zumindest in der Hauptsache um Boykottmaßnahmen, die sich gegen grenzüberschreitende Marktaktivitäten eines Dritten richteten. ${ }^{4}$

\section{Fehlende Verankerung in den gemeinsamen Verfassungstraditionen der Mitglied-} staaten $^{5}$

Trotz des Vorbehalts in Art. 137 Abs. 5 des EG-Vertrags, der von der Regelungskompetenz der Gemeinschaft ua. das Koalitionsrecht, das Streikrecht und das Aussperrungsrecht ausnimmt, hat der Europäische Gerichtshof das Recht auf Durchführung einer kollektiven Maßnahme einschließlich des Streikrechts als Grundrecht anerkannt, welches fester Bestandteil der allgemeinen Grundsätze des Gemeinschaftsrechts sei. ${ }^{6}$

Der Gerichtshof hat in der Vergangenheit ungeschriebene Gemeinschaftsgrundrechte aus den gemeinsamen Verfassungstraditionen der Mitgliedstaaten und den völkerrechtlichen Verträgen insbesondere der EMRK abgeleitet. Dies beruht letztlich auf Art. 6 Abs. 2 des Vertrags über die Europäische Union, mit dem die vorangegangene Rechtsprechung des Gerichtshofs zur Herleitung von Gemeinschaftsgrundrechten übernommen worden ist. ${ }^{7}$ Von diesen Vorgaben scheint sich der Gerichtshof in seinen Urteilen in den Rechtssachen Viking und Laval zumindest teilweise gelöst zu haben. Er hat das Grundrecht nicht auf die gemeinsamen Verfassungstraditionen der Mitgliedstaaten ge-

4 Rebhahn ZESAR 2008, 109, 114.

5 An dieser Stelle sei ausdrücklich auf die im Rahmen des Symposiums gehaltenen Ausführungen von Frau Kokott (S. 23 ff.) und Herrn Rebhahn (S. 17 ff.) verwiesen.

6 EuGH vom Dezember 2007, Viking, C-438/05, EzA GG Art. 9 Arbeitskampf Nr. 141 = NZA 2008, 124, Rn. 44.

7 EuGH 14. Mai 1974, Nold, Rs. 4/73, EuGHE 1974, 491. 
stützt, ${ }^{8}$ sondern auf vier internationale Abkommen, von denen eines - die Charta der Grundrechte der Europäischen Union - noch nicht rechtsverbindlich ist. Auch in den weiteren vom Gerichtshof angeführten Abkommen haben nicht alle Mitgliedstaaten das Streikrecht für sich als verbindlich anerkannt. ${ }^{9}$

\section{Mögliche Berücksichtigung des nationalen Rechts bei der gemeinschaftsrechtli- chen Ausgestaltung des Streikrechts}

Weil der Gerichtshof das Streikrecht ua. aus Art. 28 der Grundrechtscharta herleitet, der neben dem Gemeinschaftsrecht auf die einzelstaatlichen Rechtsvorschriften und Gepflogenheiten Bezug nimmt, geht er in den Entscheidungen Viking und Laval auf den Inhalt des finnischen 10 bzw. schwedischen ${ }^{11}$ Rechts ein und stellt fest, dass sich das Gemeinschaftsrecht bei gemeinschaftsbezogenen Arbeitskämpfen wegen des jeweils im nationalen Recht vorhandenen Vorbehalts durchsetzt. Dass man einen solchen Vorbehalt auch für das deutsche Recht anerkennen muss, kann vom Ergebnis her schwerlich in Frage gestellt werden. ${ }^{12}$ Allerdings ist der Gerichtshof - stärker als bisher erkennbar - gehalten, bei der Ausgestaltung des Grundrechts auf kollektive Maßnahmen dem Arbeitskampfrecht des jeweiligen Mitgliedstaats einen größeren Einfluss einzuräumen. Dies folgt aus dem in Art. 137 Abs. 5 EG ausdrücklichen enthaltenen Vorbehalt zu Gunsten der Mitgliedstaaten. ${ }^{13}$

8 Der dabei gebotene Rekurs auf die Ausgestaltung des Arbeitskampfrechts in den Mitgliedstaaten hätte möglicherweise auch keinen gemeinschaftsweit anerkannten Mindestanstandart ergeben, auf dessen Grundlage ein Gemeinschaftsgrundrecht hätte hergeleitet werden können.

9 Dazu ausführlich Rebhahn ZESAR 2008, 109, 110 ff.; die Herleitung und die inhaltliche Ausgestaltung eines so bedeutenden Gemeinschaftsgrundrechts, dass sich nicht mehr - und insbesondere nicht in der vom Gerichtshof gegebenen Form - an gemeinsamen Verfassungstraditionen der Mitgliedstaaten und den von ihnen abgeschlossenen völkerrechtlichen Verträgen orientiert, wird - wie schon die Herleitung des Grundsatzes der Gleichbehandlung in Beschäftigung und Beruf - die Frage nach einer entsprechenden Kompetenz des Gerichtshofs aufwerfen.

10 EuGH vom 11. Dezember 2007, Viking, C-438/05, EzA GG Art. 9 Arbeitskampf Nr. 141 = NZA 2008, 124, Rn. 44: „Außerdem kann das Streikrecht, wie aus Randnr. 5 des vorliegenden Urteils hervorgeht, nach finnischem Recht u. a. dann nicht ausgeübt werden, wenn der Streik gegen die guten Sitten, das innerstaatliche Recht oder das Gemeinschaftsrecht verstoßen würde.“

11 EuGH vom 18. Dezember 2007, Laval, C-341/05, EzA GG Art. 9 Arbeitskampf Nr. 142 = NZA 2008, 159, Rn. 92: „Es trifft zwar zu, wie die schwedische Regierung in Erinnerung ruft, dass das Recht auf Durchführung kollektiver Maßnahmen in Schweden ebenso wie in anderen Mitgliedstaaten den Schutz der Verfassung genießt, doch ändert dies nichts daran, dass, wie sich aus Randnr. 10 des vorliegenden Urteils ergibt, dieses Recht, das in diesem Mitgliedstaat die Blockade von Baustellen umfasst, nach der schwedischen Verfassung ausgeübt werden kann, wenn gesetzliche oder vertragliche Bestimmungen dem nicht entgegenstehen."

12 Dafür auch Zwanziger, DB 2008, 294, 295, der Art. 23 Abs. 1 GG als ausreichende Ermächtigungsnorm ansieht.

13 Im Rahmen meines Vortrags habe ich hierzu die Frage nach der dogmatischen Verankerung des Vorbehalts und seiner Existenz im nationalen Recht aufgeworfen. 
4. Widersprüche in der Ausgestaltung des Streikrechts durch den europäischen Gerichtshof gegenüber dem nationalem Recht

Nach der Entscheidung des Gerichtshofs in der Rechtssache Viking ist die durch das Grundrecht auf kollektive Maßnahmen geschützte gemeinschaftsrechtliche Position teilweise weiter gehend als nach nationalem Recht. Der Gerichtshof hat weder die Übernahme der Arbeitskampfmaßnahmen durch eine Gewerkschaft gefordert noch hervorgehoben, dass die kollektiven Maßnahmen auf ein tariflich regelbares Ziel gerichtet sein müssen. Daher müssten - anders als nach nationalem Recht - auch ein wilder, d.h. gewerkschaftlich nicht getragener Streik und andere kollektive Maßnahmen zur Verbesserung der Arbeitsbedingungen wie zB. die Ausübung eines kollektiven Zurückbehaltungsrechts oder ein kollektiver Widerspruch gegen einen Betriebsübergang von dem Gemeinschaftsgrundrecht geschützt sein. Gleiches könnte für einen reinen Sympathieoder einen politischen Streik gelten.

Insoweit ist die Anerkennung des Streikrechts für die Verbände durchaus ambivalent: die Ausgestaltung des Arbeitskampfrechts durch Art. 9 Abs. 3 GG schützt den Arbeitgeber vor unzulässigen Kampfformen und -mitteln der Gewerkschaft. Er müsste aber einen auf ein tariflich nicht regelbares Ziel gerichteten Arbeitskampf hinnehmen, wenn der Arbeitskampf wegen der gewerkschaftlichen Forderung in den Anwendungsbereich des Gemeinschaftsrechts fällt und die geltend gemachte Forderung den Anforderungen des gemeinschaftsrechtlichen Verhältnismäßigkeitsprinzips genügt.

$\mathrm{Zu}$ diesen Friktionen gegenüber dem nationalen Recht kommt eine weitere Problematik hinzu, die der dogmatischen Herleitung des Streikrechts geschuldet ist. Art. 28 der Grundrechtscharta gewährt das Recht auf Kollektivmaßnahmen ua. nach den einzelstaatlichen Rechtsvorschriften und Gepflogenheiten. Wird daher das Streikrecht bei gemeinschaftsrechtsrelevanten Arbeitskämpfen einer doppelten Schranke unterworfen? Müssen die kämpfenden Verbände bei der Wahrnehmung des „Grundrechts“ danach sowohl die gemeinschaftsrechtlichen Vorgaben als auch die des nationalen Rechts beachten?

\section{Die Abwägung zwischen Grundfreiheiten und Grundrechten}

$\mathrm{Zu}$ den zu Recht als problematisch angesehenen Teilen der Entscheidung Viking gehört das Verhältnis der Grundfreiheiten zu den Gemeinschaftsgrundrechten. Dabei wählt der Gerichtshof bei seiner Prüfung einen gegenüber seiner Entscheidung in der Rechtssache Schmidberger veränderten Ansatz. Dort hatte er noch ausgeführt, dass die Anwendung der dort betroffenen Grundrechte der Meinungs- und Versammlungsfreiheit mit den Erfordernissen hinsichtlich der durch den Vertrag geschützten Rechte in 
Einklang gebracht werden und dem Verhältnismäßigkeitsgrundsatz entsprechen muss. ${ }^{14}$ Dies konnte als Anzeichen für die Gleichrangigkeit der widerstreitenden Interessen angesehen werden. Ähnliche Formulierungen verwendet er zwar auch in der Rechtssache Viking, ${ }^{15}$ eine Einschränkung der Grundfreiheiten im Lichte der Grundrechte und eine Abwägung zwischen „wirtschaftlichen Freiheiten“ und „sozialen Grundrechten“ im Sinne einer praktischen Konkordanz unterbleibt jedoch. ${ }^{16}$ Ein solcher Ansatz zu Gunsten der Grundfreiheiten wirkt sich jedoch notwendigerweise auf die inhaltliche Prüfung des Eingriffs und ihr Ergebnis aus. Ist die Tarifforderungen erhebende Arbeitnehmerseite bei einem Arbeitskampf in Zukunft nach Europarecht stets gezwungen, ihre Forderungen und Kampfmittel - ggf. vor den Arbeitsgerichten - zu rechtfertigen?

\section{Die Prüfung unter dem Gesichtspunkt der Verhältnismäßigkeit}

Steht fest, dass der Arbeitskampf in eine oder vielleicht sogar mehrere Grundfreiheiten eingreift, ist nach der Rechtsprechung des Gerichtshofs über die „Rechtfertigung“ dieses Eingriffs nach Verhältnismäßigkeitsgesichtspunkten zu entscheiden. Die Rechte aus den Grundfreiheiten müssen gegen die mit der Sozialpolitik verfolgten Ziele abgewogen werden. ${ }^{17} \mathrm{Im}$ Rahmen dieser Abwägung wird vom Gerichtshof der Inhalt des Gemeinschaftsgrundrechts nicht mehr erwähnt. Diese Beurteilung obliegt dem nationalen Gericht, das ,allein für die Beurteilung des Sachverhalts und die Auslegung der nationalen Rechtsvorschriften zuständig ist, zu bestimmen, ob und inwieweit die genannte kollektive Maßnahme diesen Anforderungen entspricht". 18

Für die Prüfung der Verhältnismäßigkeit macht der Gerichtshof dem nationalen Gericht in der Entscheidung Viking nur wenige Vorgaben, lediglich dass es an einem legitimen Ziel für die Arbeitskampfmaßnahmen fehlt, wenn ,die fraglichen Arbeitsplätze oder Arbeitsbedingungen nicht gefährdet oder ernstlich bedroht waren“. ${ }^{19}$ Diese Ein-

14 EuGH vom 12. Juni 2003, Schmidberger, C-112/00, EuGHE I 2003, 5659, Rn. 74.

15 EuGH vom 11. Dezember 2007, Viking, C-438/05, EzA GG Art. 9 Arbeitskampf Nr. 141 = NZA 2008, 124, Rn. 79.

16 Blanke, Die Entscheidungen des EuGH in den Fällen Viking, Laval und Rueffert - Domestizierung des Streikrechts und europaweite Nivellierung der industriellen Beziehungen, in: Oldenburger Studien zur Europäisierung und zur transnationalen Regulierung, S. 12, abzurufen unter http://www.uni- oldenburg.de/cetro/31251.htm; Kocher ArbuR 2008, 13, 14 f.; Zwanziger, DB 2008, 294, $295 \mathrm{f}$.

17 EuGH vom 18. Dezember 2007, Laval, C-341/05, EzA GG Art. 9 Arbeitskampf Nr. 142 = NZA 2008, 159, Rn. 104 f.; 11. Dezember 2007, Viking, C-438/05, EzA GG Art. 9 Arbeitskampf Nr. 141 $=$ NZA 2008, 124, Rn. $78 \mathrm{f}$.

18 EuGH 11. Dezember 2007, Viking, C-438/05, EzA GG Art. 9 Arbeitskampf Nr. $141=$ NZA 2008, 124, Rn. 85.

19 EuGH 11. Dezember 2007, Viking, C-438/05, EzA GG Art. 9 Arbeitskampf Nr. $141=$ NZA 2008, 124, Rn. 81. 
schränkung ist für Tarifauseinandersetzungen um bessere Arbeitsbedingungen oder den Erhalt von gefährdeten Arbeitsplätzen nicht von Bedeutung.

\section{Die Konkurrenzsituation zwischen Gemeinschaftsrecht und nationalem Recht}

Nach den Entscheidungen Viking und Laval wird zukünftig zwischen Arbeitskämpfen zu differenzieren sein, in denen die Grundfreiheiten der betroffenen Unternehmen beeinträchtigt werden und die deshalb in den Anwendungsbereich des Gemeinschaftsrechts fallen und solchen ohne Gemeinschaftsbezug, die sich wie bisher nach nationalem Recht beurteilen. Maßgeblich ist danach, ob auf Seiten der betroffenen Unternehmen durch die erhobenen Forderungen in die Grundfreiheiten des EG-Vertrags eingegriffen werden soll.

Als solche kommen nach der Entscheidung des Gerichtshofs insbesondere die Dienstleistungsfreiheit (Art. 49 EG), die Niederlassungsfreiheit (Art. 43 EG) und wohl auch die Warenverkehrsfreiheit des Art. 28 EG in Betracht. ${ }^{20}$ Die Niederlassungsfreiheit (Art. 43 EG) wird nach der Rechtsprechung des Gerichtshofs beeinträchtigt, wenn es sich um eine kollektive Maßnahme handelt, die im Fall ihrer Durchsetzung geeignet wäre, es für das betroffene Unternehmen weniger attraktiv und sogar zwecklos zu machen, von seiner Niederlassungsfreiheit Gebrauch zu machen. ${ }^{21}$ Die Dienstleistungsfreiheit des Art. 49 EG ist betroffen, wenn die geforderten Arbeitsbedingungen für die entsandten Arbeitnehmer über die in dem anderen Mitgliedstaat für die dort beschäftigten Arbeitnehmer geltenden Mindestarbeitsbedingungen hinausgehen. ${ }^{22}$

Zumindest die vom Gerichtshof verwandte Formulierung des Eingriffs in die Niederlassungsfreiheit erlaubt keine trennscharfe Abgrenzung von Arbeitskämpfen, die sich nach Gemeinschaftsrecht bzw. nach nationalem Recht beurteilen. So dürfte die Niederlassungsfreiheit noch nicht bei einer gewerkschaftlichen Forderung auf Abschluss eines Tarifsozialplans betroffen sein, der die Verlagerung eines Unternehmens in einen anderen Mitgliedstaat „verteuert“. ${ }^{23}$ Eindeutig ist das aber nicht. Ebenso bleibt fraglich, ob eine gegenüber einer ausländischen Gesellschaft mit Sitz in einem anderen Mitgliedstaat oder ihrer deutschen Tochter erhobene Tarifforderung zu einem Gemeinschaftsbezug führt, weil der mit einem Tarifvertragsabschluss verbundene Anstieg der Personalkosten die Attraktivität des hiesigen Standorts schmälert.

Ist für ein nationales Arbeitsgericht zweifelhaft, ob ein Arbeitskampf, dessen Rechtmäßigkeit es zu beurteilen hat, in den Anwendungsbereich des Gemeinschaftsrechts

20 Dazu Rebhahn, ZESAR 2008, 109, 116 f.

21 EuGH vom 11. Dezember 2007, Viking, C-438/05, EzA GG Art. 9 Arbeitskampf Nr. 141 = NZA 2008, 124, Rn. 72.

22 EuGH 18. Dezember 2007, Laval, C-341/05, EzA GG Art. 9 Arbeitskampf Nr. 142 = NZA 2008, 159, Rn. 99.

23 Ebenso Rebhahn, ZESAR 2008, 109, 115; anders wohl Temming, ZESAR 2008, 231, 241. 
fällt, ist es im Rahmen des Art. 243 EG berechtigt bzw. verpflichtet, über die Reichweite der Grundfreiheiten den Gerichtshof im Rahmen eines Vorabentscheidungsverfahrens anzurufen. Dieses könnte zB. die Frage betreffen, ob ein Streik bei Eisenbahngesellschaften die Warenverkehrsfreiheit beeinträchtigt oder auch ein politischer Streik von dem Grundrecht auf kollektive Maßnahmen geschützt wird.

Korrigiert der Gerichtshof seine umstrittene Dogmatik zum Verhältnis von Grundfreiheiten und „Grundrechten“ nicht, so bleibt für die Berücksichtigung der bisherigen Grundsätze des nationalen Arbeitskampfrechts nur die Verhältnismäßigkeitsprüfung im Rahmen der Rechtfertigung des Eingriffs in die Grundfreiheiten. An dieser Stelle ist spätestens - der Vorbehalt des Art. 137 Abs. 5 EG von Bedeutung. Dieser ermöglicht den nationalen Gerichten eine gemeinschaftsrechtsgeleitete Prüfung unter Heranziehung der in der Vergangenheit von Bundesverfassungsgericht und Bundesarbeitsgericht entwickelten Grundsätze. Die gerichtliche Kontrolle des Umfangs der erhobenen Tarifforderungen oder die Wahl der zu ihrer Durchsetzung eingesetzten Mittel würde danach von Extremfällen abgesehen - auch zukünftig unterbleiben.

\section{Dritte These}

Der Europäische Gerichtshof sollte in Entscheidungen, die das Recht der Mitgliedstaaten erstmals dem Anwendungsbereich des Gemeinschaftsrechts unterwerfen, einen nachvollziehbaren Begründungsansatz wählen. ${ }^{24}$

Die teilweise auf die Mitteilung des Ergebnisses und eine aus sich heraus nur schwer nachvollziehbare Begründung beschränkte Darstellung der Entscheidungen des Gerichtshofs stellen die nationalen Gerichte vor Probleme. Eine etwas ausführlicher begründete Entscheidung des Gerichtshofs würde vielfach ihre Akzeptanz erhöhen und erneute Vorabentscheidungsverfahren vermeiden, die für alle an einem solchen Verfahren beteiligten Personen und Stellen einen beträchtlichen Aufwand bedeuten. Dies gilt insbesondere, wenn der Gerichtshof den Anwendungsbereich des Gemeinschaftsrechts ausweitet oder neue und ausfüllungsbedürftige Rechtsgrundsätze aufstellt. Ansonsten läuft die betreffende Entscheidung des Gerichtshofs Gefahr, von den Gerichten der Mitgliedstaaten nicht oder nicht zutreffend gewürdigt zu werden. Dazu zwei aktuelle Beispiele:

24 Auch dieser Teil konnte aus Zeitgründen nicht mündlich vorgetragen werden. 


\section{Die Entscheidung Werhof}

Das Vorabentscheidungsersuchen des Landesarbeitsgerichts Düsseldorfs betraf kurz zusammengefasst - die Auslegung einer vertraglichen Bezugnahmeklausel auf einen Tarifvertrag im Zusammenhang mit einem Betriebsübergang. In diesem Zusammenhang führte der Gerichtshof sinngemäß und ohne weitere Begründung aus, dass eine Auslegung der vertraglichen Verweisklausel als „dynamisch“ für den Betriebserwerber zu einer Beeinträchtigung seines Grundrechts auf negative Koalitionsfreiheit führen könnte. ${ }^{25}$ Die Vorstellung von einem Verstoß gegen die negative Koalitionsfreiheit des Arbeitgebers durch Bindung eben dieses Arbeitgebers an den von ihm vorformulierten Arbeitsvertrag erscheint - nicht nur mir - nicht mehr nachvollziehbar. Möglicherweise hat der Gerichtshof bei seiner Entscheidungsfindung nicht hinreichend bedacht, dass es im Streitfall nicht um eine besondere Form von Tarifbindung, sondern schlicht um Vertragsfreiheit geht. Dass diese Verpflichtung aus dem Arbeitsvertrag dann auch den Betriebserwerber trifft, ist Folge des Art. 3 Abs. 1 der Betriebsübergangsrichtlinie. So stehen weitere Nachfragen der deutschen Gerichte zu erwarten.

\section{Der Super-GAU - der Konflikt zwischen Gemeinschafts- und nationalem}

Verfassungsrecht

Die gleichermaßen in der Begründung knapp abgefasste Entscheidung des Gerichtshofs in der Rechtssache Mangold ist auf breite Kritik gestoßen, die dem Gerichtshof ua. eine Überschreitung seiner Kompetenzen vorwarf und sich für die Unverbindlichkeit des Unanwendbarkeitsausspruchs aussprach. ${ }^{26}$ Der Siebte Senat des Bundesarbeitsgerichts ist dem nicht gefolgt. Er hat versucht, die Entscheidungsgründe seinerseits auszulegen und hat angenommen, dass der Gerichtshof die ihm durch die deutschen Zustimmungsgesetze zu den Europäischen Verträgen übertragenen Kompetenzen nicht überschritten hat. ${ }^{27}$ Vor der Entscheidung des Bundesarbeitsgerichts ist diskutiert worden, ob der erkennende Senat den Europäischen Gerichthof erneut anzurufen sollte, um ihn Gelegenheit zur Ergänzung seines primärrechtlichen Prüfungsansatzes zu geben. Bei einer etwas breiteren und nachvollziehbareren Darstellung des primärrechtlichen Prüfungsansatzes wäre diese Diskussion sicherlich nicht oder nicht in dieser Schärfe geführt worden. Gegen diese Entscheidung des Bundesarbeitsgerichts hat die unterlegene Arbeitgeberin Verfassungsbeschwerde beim Bundesverfassungsgericht eingelegt. ${ }^{28}$ Ich

25 EuGH vom 9. März 2006, Werhof, C-449/04, EuGHE I 2006, 2397 = AP Richtlinie 77/187/EWG Nr. 2 = EzA BGB $2002 \S 613$ a Nr. 44, Rn. 34.

26 Bauer/Arnold, NJW 2006, 6, 8; wohl auch Nicolai,DB 2005, 2641, 2642.

27 BAG 26. April 2006, 7 AZR 500/04, BAGE 118, $76=$ AP TzBfG $\S 14$ Nr. $23=$ EzA TzBfG $\S 14$ Nr. 28, Rn. 17.

28 Az. 2 BvR 2661/06. 
bin mir bis heute nicht im Klaren darüber, welchen Inhalt ein erneutes Vorabentscheidungsverfahren hätte haben können. Über die Frage, ob der Europäische Gerichtshof eine von den deutschen Zustimmungsgesetzen zu den Europäischen Verträgen nicht mehr gedeckte Rechtsfortbildung vorgenommen hat, kann nach meinem Dafürhalten wenn überhaupt - nur das Bundesverfassungsgericht verbindlich entscheiden. Art. 100 des Grundgesetzes lässt aber nach seinem Wortlaut eine so begründete Richtervorlage an das Bundesverfassungsgericht nicht zu. Dieser Zustand ist aus meiner Sicht unbefriedigend.

Möglicherweise hat das Bundesverfassungsgericht bei seiner Entscheidung über die gegen die Entscheidung des Siebten Senats eingelegte Verfassungsbeschwerde Veranlassung, für die Konfliktfälle bei der Abgrenzung von Gemeinschaftsrecht und nationalem Recht klare Vorgaben aufzustellen. Dann allerdings wäre der fragwürdige befristete Arbeitsvertrag zwischen den Rechtsanwälten Werner Mangold und Rüdiger Helm nicht nur durch die denkwürdige Entscheidung des Europäischen Gerichtshofs, sondern auch noch durch eine Entscheidung des Bundesverfassungsgerichts, durch die das Verhältnis zwischen Gemeinschaftsrecht und nationalem Recht austariert würde, ein weiteres Mal geadelt. 
